\title{
Intratumoral immunotherapy with STING agonist, ADU-S100, induces CD8+ T-cell mediated anti-tumor immunity in an esophageal adenocarcinoma model
}

\author{
Ali H. Zaidi ${ }^{1,}{ }^{*}$, Ronan J. Kelly ${ }^{2,}{ }^{*}$, Anastasia Gorbunova ${ }^{1}$, Ashten N. Omstead ${ }^{1}$, \\ Madison S. Salvitti ${ }^{1}$, Ping Zheng ${ }^{1}$, Juliann E. Kosovec ${ }^{1}$, Soyoung Lee $^{3}$, Shahin \\ Ayazi $^{1}$, Laila Babar ${ }^{1}$, Gene G. Finley ${ }^{1}$, Ajay Goel ${ }^{4}$ and Blair A. Jobe ${ }^{1}$ \\ ${ }^{1}$ Esophageal and Lung Institute, Allegheny Health Network, Pittsburgh, PA, USA \\ ${ }^{2}$ Department of Hematology and Oncology, Charles A. Sammons Cancer Center, Baylor University Medical Center, Dallas, \\ TX, USA \\ ${ }^{3}$ Department of Radiation Oncology, Allegheny Health Network, Pittsburgh, PA, USA \\ ${ }^{4}$ Department of Molecular Diagnostics and Experimental Therapeutics, Beckman Research Institute of City of Hope, Monrovia, \\ CA, USA \\ ${ }^{*}$ Co-first authors and contributed equally to this work \\ Correspondence to: Ali H. Zaidi, email: Ali.zaidi@ahn.org \\ Keywords: esophageal adenocarcinoma; STING; PD-L 1; CD8+ T-cells; IFNß \\ Received: November 20, $2020 \quad$ Accepted: January 26, $2021 \quad$ Published: February 16, 2021
}

Copyright: ( 2021 Zaidi et al. This is an open access article distributed under the terms of the Creative Commons Attribution License (CC BY 3.0), which permits unrestricted use, distribution, and reproduction in any medium, provided the original author and source are credited.

\section{ABSTRACT}

Background: Esophageal adenocarcinoma (EAC) is a deadly disease with limited treatment options. STING is a transmembrane protein that activates transcription of interferon genes, resulting in stimulation of APCs and enhanced CD8+ T-cell infiltration. The present study evaluates STING agonists, alone and in combination with radiation to determine durable anticancer activity in solid tumors.

Materials and Methods: Esophagojejunostomy was performed on rats to induce reflux leading to the development of EAC. At 32 weeks post operatively, rats received intratumorally either $50 \mathrm{\mu g}$ STING (ADU-S100) or placebo (PBS), +/- 16Gy radiation. Drug activity was evaluated by pre- and post- treatment MRI, histology, immunofluorescence and RT-PCR.

Results: Mean MRI tumor volume decreased by $\mathbf{3 0 . 1 \%}$ and $\mathbf{5 0 . 8 \%}$ in ADU-S100 and ADU-S100 + radiation animals and increased by $76.7 \%$ and $152.4 \%$ in placebo and placebo + radiation animals, respectively $(P<0.0001)$. Downstream gene expression, pre- to on- and post- treatment, demonstrated significant upregulation of IFN $\beta$, TNFa, IL-6, and CCL-2 in the treatment groups vs. placebo. On- or posttreatment, radiation alone, ADU-S100 alone, and ADU-S100 + radiation groups demonstrated enhanced PD-LI expression, induced by upregulation of CD8 + T-cells $(p<0.01)$.

Conclusions: ADU-S100 +/- radiation exhibits potent antitumor activity and a promising immunomodulatory profile in a de novo EAC.

\section{INTRODUCTION}

Esophageal cancer is the sixth most common cause of cancer related deaths worldwide [1]. Currently, the mainstay of treatment consists of chemotherapy, radiation, surgery, PD-1/PD-L1 checkpoint blockade in microsatellite instability high (MSI-H) tumors, and targeted therapy with monoclonal antibodies targeting HER2 [2]. Despite these therapeutic modalities, overall survival in stage IV patients remains poor with a 5 year survival rate of less than $20 \%$ [3]. Therefore, new advancements to enhance therapeutics efficacy are much needed.

Targeting the PD-1/PD-L1 axis has proven to be highly efficacious in patients with advanced solid tumors 
including melanoma, renal cell carcinoma, and non-small cell lung cancer $[4,5]$. Durable responses to ICIs are typically observed in the presence of tumor-infiltrating T-cells (TIL) [6]. In gastroesophageal cancers PD-L1 upregulation occurs in approximately $40 \%$ of patients, predominantly on infiltrating myeloid cells at the invasive margin rather than cancer cells $[7,8]$. However, initial results of ICIs in the treatment of esophageal cancer (EC) have been marginal with encouraging signals mostly limited to esophageal squamous cell carcinoma (ESCC), MSI-H, and subgroups with combined positivity score $(\mathrm{CPS})>10$ [2]. Therefore, a future promising strategy to enhance efficacy of ICIs in non-immunogenic cold tumors, such as esophageal cancer, is to promote T-cell infiltration by activation of the innate immune system [9].

The key regulator of the innate immune system is the stimulator of interferon genes (STING), an endoplasmic reticulum adaptor protein, which stimulates the production of type 1 interferons (IFN) from cancer cells and dendritic cells (DC) in the tumor microenvironment. Briefly, the tumor or pathogen derived cytosolic DNA in these cells is recognized by cytosolic enzyme cGAMP synthase leading to the generation of cyclic GMP-AMP, which in turn binds to and activates STING signaling. This initiates a cascade where STING in the cytoplasm binds to TANK-binding kinase 1 (TBK1) and I $\mathrm{B}$ kinase (IKK), that activates a host of transcription factors: interferon regulatory factor 3 (IRF3), nuclear factor- $\kappa \mathrm{B}(\mathrm{NF}-\kappa \mathrm{B})$, and signal transducer and activator of transcription (STAT6). Subsequently, nuclear translocation of these transcription factors leads to the induction of type I IFNs and other immune modulatory cytokines.[10, 11] Type 1 IFNs have multiple immunestimulatory effects that include activation, maturation, and migration of multiple immune cells including natural killer (NK) cells, DCs and anti-tumor T-cells [12].

Therefore, as expected, in preclinical models STING agonists have demonstrated not only potent activity against the targeted primary tumors but also for distant metastasis and recurrence $[13,14]$. Additionally, combination of STING agonists to standard of care radiation and immunotherapy have demonstrated enhanced antitumor activity $[15,16]$. Specifically, Deng et al demonstrated in mice that damaged double stranded DNA from irradiated cells leads to enhanced activity of STING pathway in DCs to promote radiation-induced type I IFN immune response [17].

In this study we evaluate the impact of a STING agonist, ADU-S100, a synthetic cyclic dinucleotide (CDN) agonist of STING, known to activate all human and mouse STINGs and induce the expression of cytokines and chemokines [18], in combination with radiation, on local tumor control and effector T-cell functionality using the modified Levrat model for esophageal adenocarcinoma (EAC). This surgical model of end-to-side esophagojejunostomy in rats causes chronic gastroduodenoesophageal reflux disease (GDER) inducing the development of de novo EAC through identical physiological and molecular processes that occur in humans $[19,20]$. Additionally, the model offers an intact immune system that on exposure to radiation has demonstrated enhanced PD-L1 sensitization that is dose dependent and transient in nature [21]. This provides an ideal translatable EAC model to study synergistic efficacy and immunomodulation profiles on combining STING agonists with radiation.

\section{RESULTS}

Thirty weeks after the Modified levrat surgery was performed, 85 rats were randomized to the four intervention groups: placebo $(\mathrm{P})(n=20)$, placebo with radiation $(\mathrm{P}+\mathrm{R})(n=16), \mathrm{STING}$ agonist $(\mathrm{S})(n=29)$ and $\mathrm{STING}$ agonist with radiation $(\mathrm{S}+\mathrm{R})(n=20)$. The overall mortality rate post randomization was $20 \%(n=17)$, with the S and S+R treatment cohort representing $64.7 \%$ of the total mortality $(n=11)$. Causes of mortality within the control groups $(\mathrm{P}$ and $\mathrm{P}+\mathrm{R})$ included respiratory infection secondary to aspiration of reflux $(n=2)$, strictures at the anastomotic site $(n=2)$, perforated stomach ulcers $(n=1)$ and unknown etiology $(n=1)$. Within the treatment groups $(\mathrm{S}$ and $\mathrm{S}+\mathrm{R})$, causes of mortality included respiratory infection secondary to aspiration $(n=6)$, strictures at the anastomotic site $(n=3)$, perforated stomach ulcers $(n=1)$ and unknown etiology $(n=1)$. There was no significant increase in observed mortality in the STING agonist treated animals $(22.5 \%)$ when compared to the controls $(16.7 \%)(p=0.59)$. Sixty-eight animals completed the study, including 30 control $(P=18$ and $\mathrm{P}+\mathrm{R}=12)$ and 38 treatment $(\mathrm{S}=23$ and $\mathrm{S}+\mathrm{R}=15)$ animals.

Overall, a comparison of MRIs in the study groups between 32 and 40 weeks demonstrated a mean increase in percentage tumor volume of $76.7 \%$ and $152.4 \%$ in the $\mathrm{P}$ and $\mathrm{P}+\mathrm{R}$ arms respectively, and a decrease of $30.1 \%$ and $50.8 \%$ in the $\mathrm{S}$ and $\mathrm{S}+\mathrm{R}$ arms, respectively (ANOVA test $p<0.0001)-$ Figure $1 \mathrm{~A}$. In the $\mathrm{P}$ and $\mathrm{P}+\mathrm{R}$ group, $76.5 \%$ and $80.0 \%$ of the rats demonstrated an increase in tumor volume, $17.6 \%$ and $10.0 \%$ had stable disease and the remaining $5.9 \%$ and $10.0 \%$ had a decrease in tumor volume, respectively. Following treatment with $\mathrm{S}$ and $\mathrm{S}+\mathrm{R}, 0.0 \%$ and $0.0 \%$ of the rats demonstrated an increase in tumor volume, $60.0 \%$ and $22.2 \%$ had stable disease and the remaining $40.0 \%$ and $77.8 \%$ had a decrease in tumor volume, respectively (Fisher's exact test $p=<0.0001$ ) Figure 1B. (Supplementary Table 1).

Analysis of STING pathway gene expression with 30-week pre-randomization biopsy RQ values set as the baseline demonstrated on- and post- $\mathrm{S}$ treatment a significant mean difference in RQ values. Similar but more moderate peak and trough changes in RQ values of downstream genes were observed in $\mathrm{S}+\mathrm{R}$ group onand post- treatment. On the contrary, $\mathrm{P}$ and $\mathrm{P}+\mathrm{R}$ groups demonstrated minor difference in on- and post- treatment 
RQ values from baseline pre- randomization biopsy levels for all the individual genes. For the individual genes, Mixed model F-test demonstrated statistically significant difference for group effect, time effect and interaction effect between group and time, respectively $(p<0.01)-$ Figure 2. (Supplementary Table 2).

TIL infiltration was upregulated with higher mean CD-8+ T-cell densities on- and post- treatment in $\mathrm{P}+\mathrm{R}$ (mean on- $=43.5, p=0.0009$; mean post- $=23.2$, $p=0.1044), \mathrm{S}$ (mean on- $=63.4, p<.0001$; mean post$=54.3, p=0.0010)$ and $\mathrm{S}+\mathrm{R}$ (mean on- $=59.8, p=$ 0.0002 ; mean post- $=42.9, p=0.0015)$ compared to $P$ (mean on- $=3.1$; mean post- $=5.1$ ). Additionally, in the drug intervention subgroups, post-hoc analysis utilizing Wilcoxon-Mann-Whitney test showed a significant difference in CD-8 positivity between pre- and both on$(p<.0001(\mathrm{~S}) ; p=0.0002(\mathrm{~S}+\mathrm{R}))$ and post- treatment $(p<.0001(\mathrm{~S}) ; p=0.0004(\mathrm{~S}+\mathrm{R}))$ time points - Figures 3 and 4. Subsequently, adaptive immune resistance depicted by total PD-L1 positive (immune stromal and tumor cells) was significantly enhanced in $\mathrm{P}+\mathrm{R}$ (mean on- = $66.1, p=0.0001$; mean post- $=35.3, p=0.0149$ ), $\mathrm{S}$ (mean on- $=72.6, p<.0001$; mean post- $=56.4, p=0.0021)$ and $\mathrm{S}+\mathrm{R}$ (mean on- $=62.5, p<.0001 ;$ mean post- $=71.0, p=$ $0.0015)$, compared to $P$ (mean on- $=2.9$; mean post- $=$ 6.3). Likewise, in the drug intervention subgroups, post-

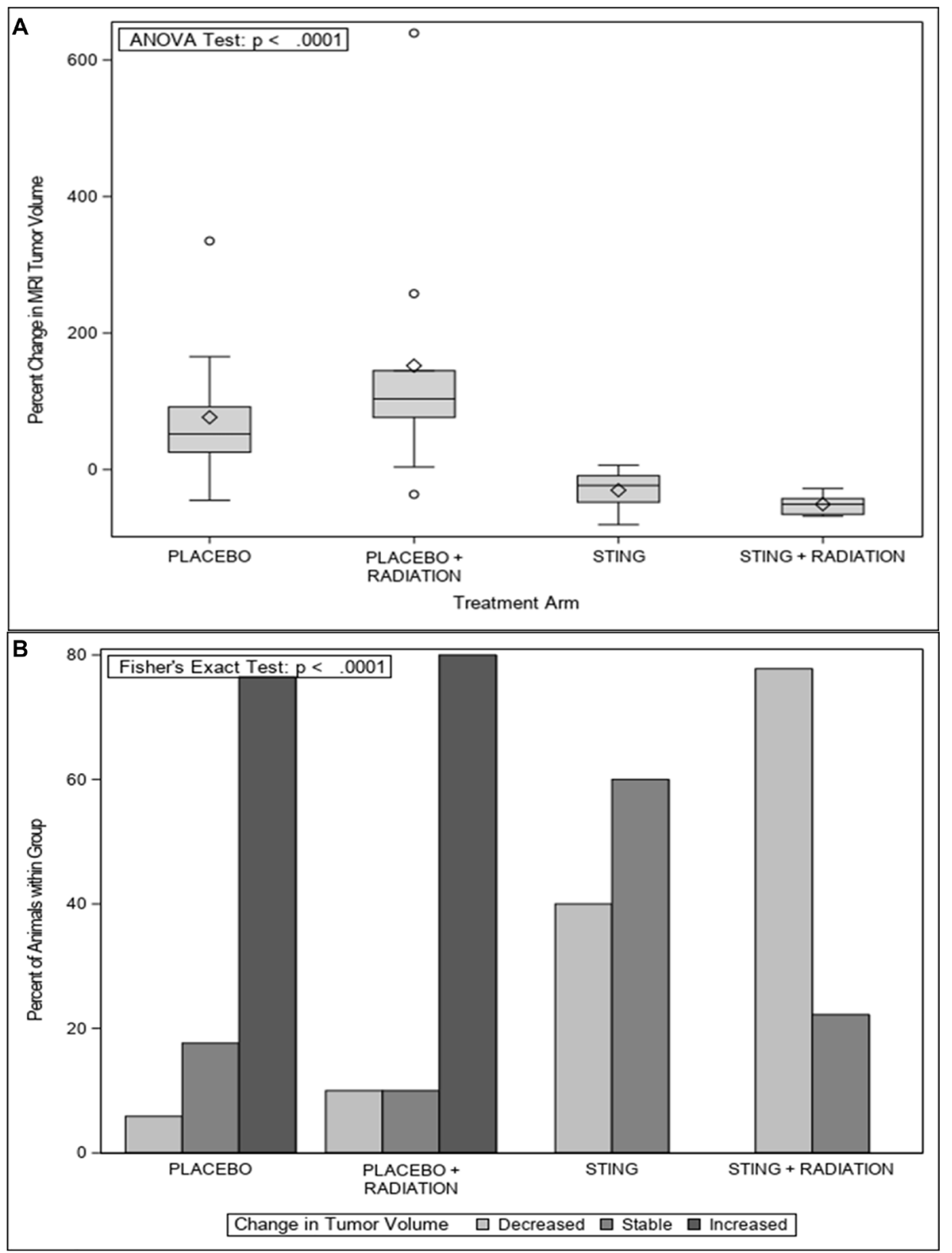

Figure 1: MRI change tumor volume, by treatment group. (A) Percent of animals with change in tumor volume as measured by MRI at 30 and 40 weeks for placebo or ADU-S100, $+/-16 \mathrm{~Gy}$ radiation groups. (B) Percent change in mean tumor volume as measured by MRI from 30 to 40 weeks for placebo or ADU-S100, +/- 16Gy radiation groups. 
hoc analysis utilizing Wilcoxon-Mann-Whitney test showed a significant difference in PD-L1 positive cells between pre- and both on- $(p<.0001(\mathrm{~S}) ; p=0.0021$ $(\mathrm{S}+\mathrm{R}))$ and post- treatment $(p=0.0003(\mathrm{~S}) ; p=0.0003$ $(\mathrm{S}+\mathrm{R}))$ time points - Figures 5 and 6 .

\section{DISCUSSION}

Activation of the STING mechanism is central for innate immune sensing which leads to the production of pro-inflammatory cytokines, especially IFN $\beta$, in the tumor microenvironment. IFN $\beta$ upregulation has been strongly correlated with T-cell priming and enhanced TIL densities thereby establishing the critical bridge between innate and adaptive immune responses [12, 23]. Overall, this study is the first report of a STING agonist demonstrating successful tumor regression in an EAC model while simultaneously unmasking favorable changes in the tumor immune microenvironment, such as enhanced TIL densities and PD-L1 expression. This charged immune microenvironment may provide future clinical opportunities for durable responses when combined with current ICI, chemotherapy, and radiation options [16, 17].

In vivo, MRI was utilized in the current study to quantify preclinical change in tumor volume in response to treatment with ADU-S100 through the comparison of 30- and 40-week scans, with each rat serving as its own control. The results demonstrated that $\mathrm{P}$ and $\mathrm{P}+\mathrm{R}$ animals exhibited $76.7 \%$ and $152.4 \%$ increase in mean tumor volume, respectively. Meanwhile, the $\mathrm{S}$ and $\mathrm{S}+\mathrm{R}$ treatment groups demonstrated greater than $30 \%$ reduction in mean tumor volume. Specifically, in the control arms $92.6 \%$ of the group either remained stable or increased in volume while $54.2 \%$ of the treatment animals had a reduction in volume. Overall, the $\mathrm{S}+\mathrm{R}$ group demonstrated the best results with maximum mean volume reduction with all cases responding. The imaging results were further validated through gene expression and evaluation of established downstream STING pathway cytokines: IFN $\beta$, TNF $\alpha$, IL-6, and CCL2 [22]. All profiled cytokines were significantly upregulated on- and post- treatment in S and $\mathrm{S}+\mathrm{R}$ groups, with the expression profiles establishing peak levels on- treatment. In the study, the in vivo gene expression levels validated the anticipated regulatory effects. While not evaluated in this study, evidence from other studies suggests that local tumor control is possibly mediated by IFN $\beta$ driven recruitment of cytotoxic CD8+ T-cells, TNF alpha mediated disruption of tumor microvasculature, and direct activation of apoptosis in cancer cells by STING signaling [12, 13, 23, 24].

ADU-S100 was given to $\mathrm{S}$ and $\mathrm{S}+\mathrm{R}$ groups as 2 cycles of $50 \mu \mathrm{g}$ intra-tumoral injections, q3 weekly. Previous data had shown this dose as immunogenic rather than tumor ablative, primarily by generating CD8+

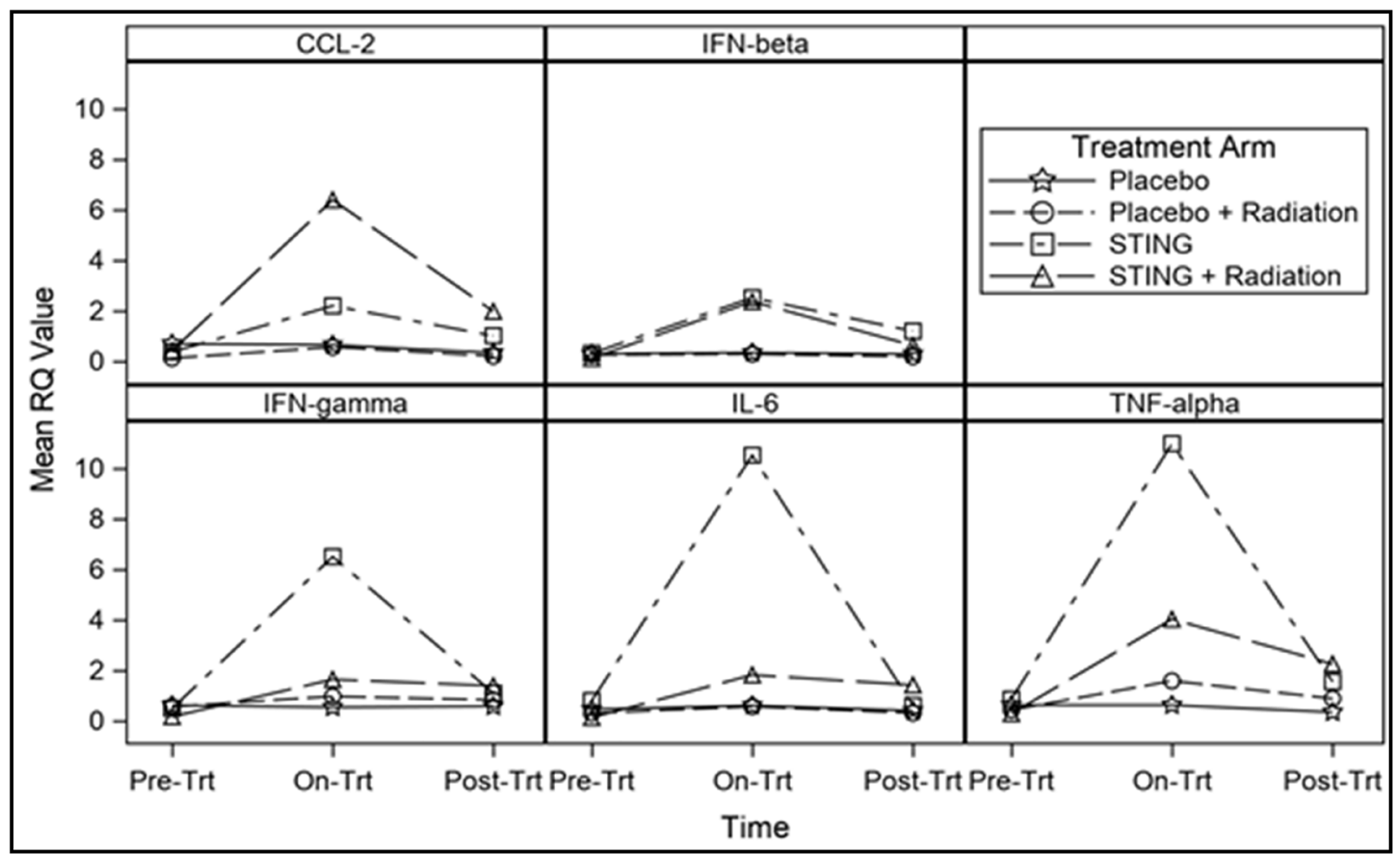

Figure 2: Pre and post treatment gene expression, by treatment group. Line charts demonstrating changes in mean RQ value for individual gene expression pre-, on- and post-intratumoral injections with placebo or ADU-S100, +/- 16Gy radiation. For all individual genes mixed model F-test demonstrated statistically significant difference for group effect, time effect and interaction effect between group and time, respectively $(p<0.01)$. 
cytotoxic T-cells for a comprehensive antitumor response through production of IFN $\beta$ via activation of STINGTBK1-IRF3 axis [25, 26]. Additionally, counterproductive vascular disruption mediated by TNF alpha for local tumor control is mostly limited at this dose selection. This is critical for generating a 'systemic' immune response to target distant metastasis and possible recurrences [25]. Our study in line with previous experiments successfully demonstrated enhanced PD-L1 expression, possibly by triggering an adaptive immune response, as suggested by increased infiltration of CD8 $+\mathrm{T}$ cells in tumors in $\mathrm{S}, \mathrm{S}+\mathrm{R}$ and $\mathrm{P}+\mathrm{R}$ groups compared to $\mathrm{P}$. These changes peaked on- treatment. Moreover, as reported previously, we demonstrated radiation and a STING agonist work additively in triggering an adaptive immune response [17], with $\mathrm{S}+\mathrm{R}$ arm showing higher densities of IFN $\gamma$ producing CD8+ T-cells when compared to radiation alone. This treatment regimen provides a significant clinical opportunity for EAC, a devastating disease with poor survival outcomes due to limited treatment options.

Overall, strategies that harness a patient's own immune system hold great promise as $40 \%$ of gastroesophageal tumors express PD-L1, mostly on infiltrating myeloid cells [8]. Single agent PD-1 inhibitors have demonstrated modest efficacy with response rates of approximately $12 \%$ in heavily pretreated gastroesophageal cancer patients, leading to clinical approvals in metastatic 3rd line setting for pembrolizumab and nivolumab (Japan only) [27, 28]. Earlier this year, based on the data from Keynote 181 [29] and Keynote 180 [30] the Food and Drug Administration approved pembrolizumab for patients with recurrent, locally advanced, or metastatic ESCC with CPS $\geq 10$, after $\geq 1$ lines of systemic therapy. Although not statistically significant, better responses were observed in patients with ESCC than EAC [2, 29]. However, the most recent readout from Keynote 062 where pembrolizumab plus chemotherapy (cisplatin with either 5-fluorouracil or capecitabine) performed worse that chemo alone in untreated advanced GEJ/Gastric cancer patients dampened the excitement around the combination of PD-1 inhibitors with chemotherapy to enhance efficacy. However, the silver lining in the study was that pembrolizumab alone was deemed non-inferior to chemotherapy alone [31].

In our opinion, to improve and expand the benefit to a majority of gastroesophageal patients, beyond the response demonstrated by single agent PD-1 inhibitors, combination with other immune oncology (IO) targets is needed. Data from CheckMate 032, has demonstrated nivolumab plus ipilimumab is superior to nivolumab alone, with ORR of $24 \%$ and $12 \%$, respectively however it is unclear if this is a viable strategy moving forwards due to enhanced toxicity with this particular IO-IO combination [32]. Currently, BMS Fraction (NCT02935634) a basket trial and Roche-Genentech Morpheus (NCT03281369) an umbrella trial are investigating multiple IO-IO or IO driven combinations in GEJ/Gastric cancers [33].
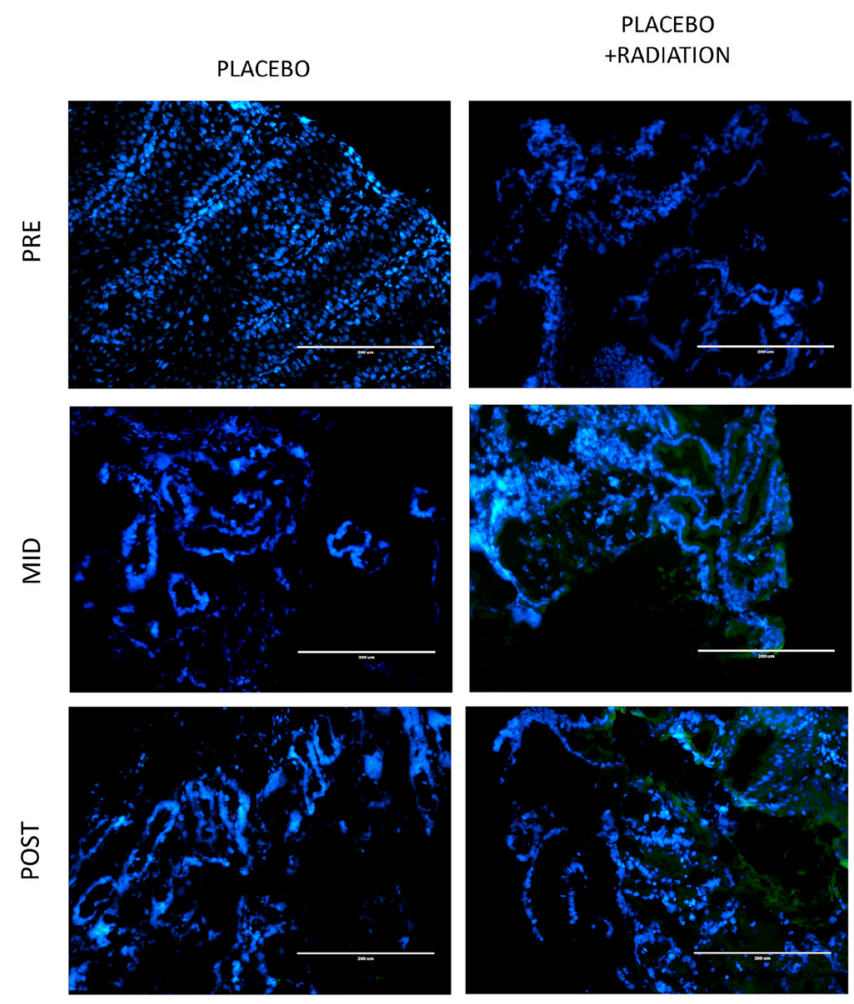
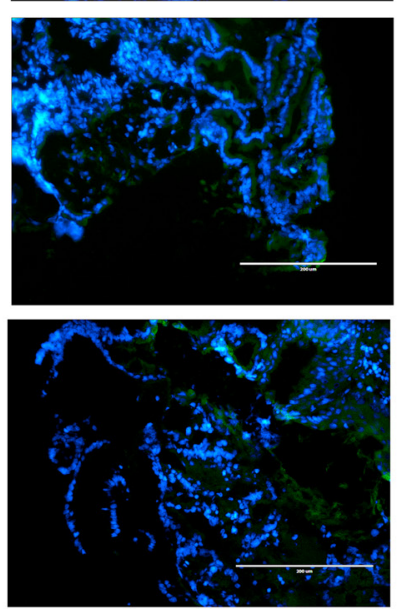

STING
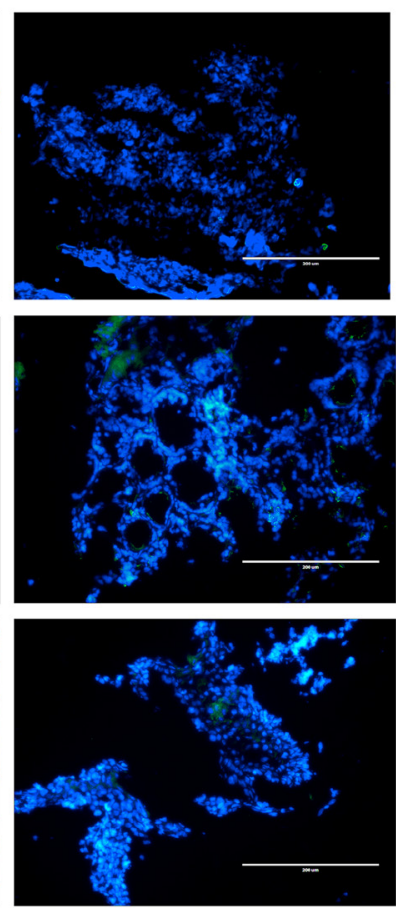

STING

+RADIATION
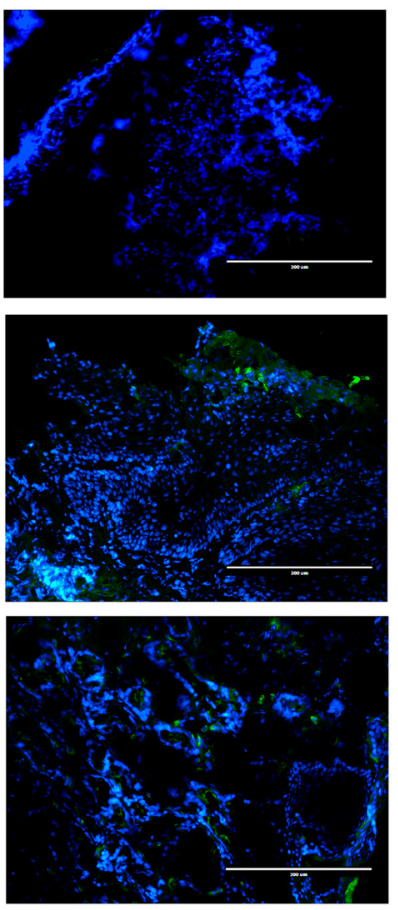

Figure 3: CD8 immunofluorescence, by treatment group. Representative study subgroup tumor samples showing membranous CD8 immunofluorescence pre-, on- and post-intratumoral injections with placebo or ADU-S100, +/- 16Gy radiation. Positive CD8 staining was detected at $20 \times$ magnification in the primary tumor with the Alexa Fluor 488 secondary antibody, conjugated to a green fluorophore. 
Through our current study, we provided strong evidence to suggest cross-link between the STING and PD-L1 pathways in EAC. A host of earlier preclinical cancer studies have also demonstrated enhanced activity of ICIs when combined with a STING agonist [16, 22, 34]. Currently, based on encouraging data, NCT03172936, NCT02675439 and NCT03937141 are studying ICIs in combination with ADU-S100 in human lymphomas and solid tumors.

The main limitation of our study was the inability to test a PD-1/PD-L1 inhibitor in combination with $\mathrm{S}$ and $\mathrm{S}+\mathrm{R}$ due to unavailability of a rat cross reactive antibody. However, we did show PD-L1 upregulation on- or posttreatment with $\mathrm{S}$ and $\mathrm{S}+\mathrm{R}$ hence substantially addressing this limitation. Another limitation may have been that we did not specifically study systemic immune response, for instance looking at peripheral T-cell trafficking. Yet previous preclinical data have well documented systemic immune modulation following a $50 \mu \mathrm{g}$ dose of ADU-S100 [25].

In conclusion, our findings suggest potent antitumor activity of ADU-S100 alone and in combination with radiation against EAC with evident molecular pathway activation and reasonable safety. Additionally, the attractive synergistic association between STING activation and PD-L1 expression may represent a new IO-IO concurrent combinatorial antitumor strategy wellsuited for further clinical testing in gastroesophageal cancers, to provide broader and more durable responses.

\section{MATERIALS AND METHODS}

\section{Animal ethics statement}

All animal research was performed with the approval of the Institutional Animal Care and Use Committee of Allegheny General Hospital in Pittsburgh, PA, USA, under Protocol \#1057. Humane care was provided to all animals per the standards set forth in "The Guide for the Care and Use of Laboratory Animals."

\section{Drug formulation and administration}

Published data was utilized to select a dose strength and frequency for ADU-S100 that was deemed highly immunostimulatory [25]. ADU-S100 was provided at a concentration of $10 \mathrm{mg} / \mathrm{mL}$ by the manufacturer (Aduro Biotech Inc., Berkeley, CA, USA), diluted with PBS to a $50 \mu \mathrm{g} / \mathrm{mL}$ concentration, and stored at $4{ }^{\circ} \mathrm{C}$. Two cycles of a $50 \mu \mathrm{g}$ dose of ADU-S100 or placebo (acetate buffer)

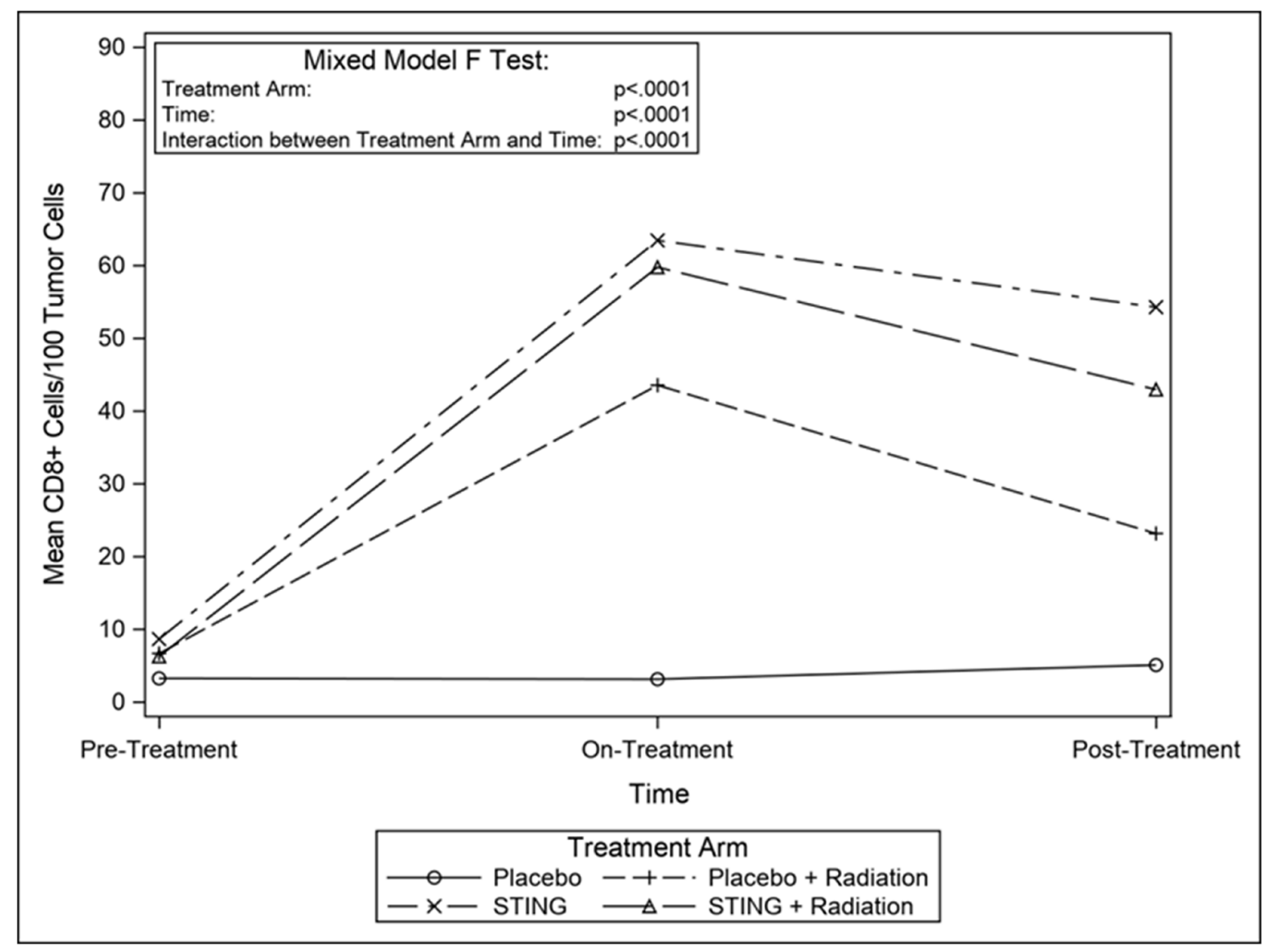

Figure 4: Mean CD8 cells/100 tumor cells, by treatment group. Line graphs depicting changes in mean number of CD8+ cells per 100 tumor cells by study groups pre- on- and post-intervention. 

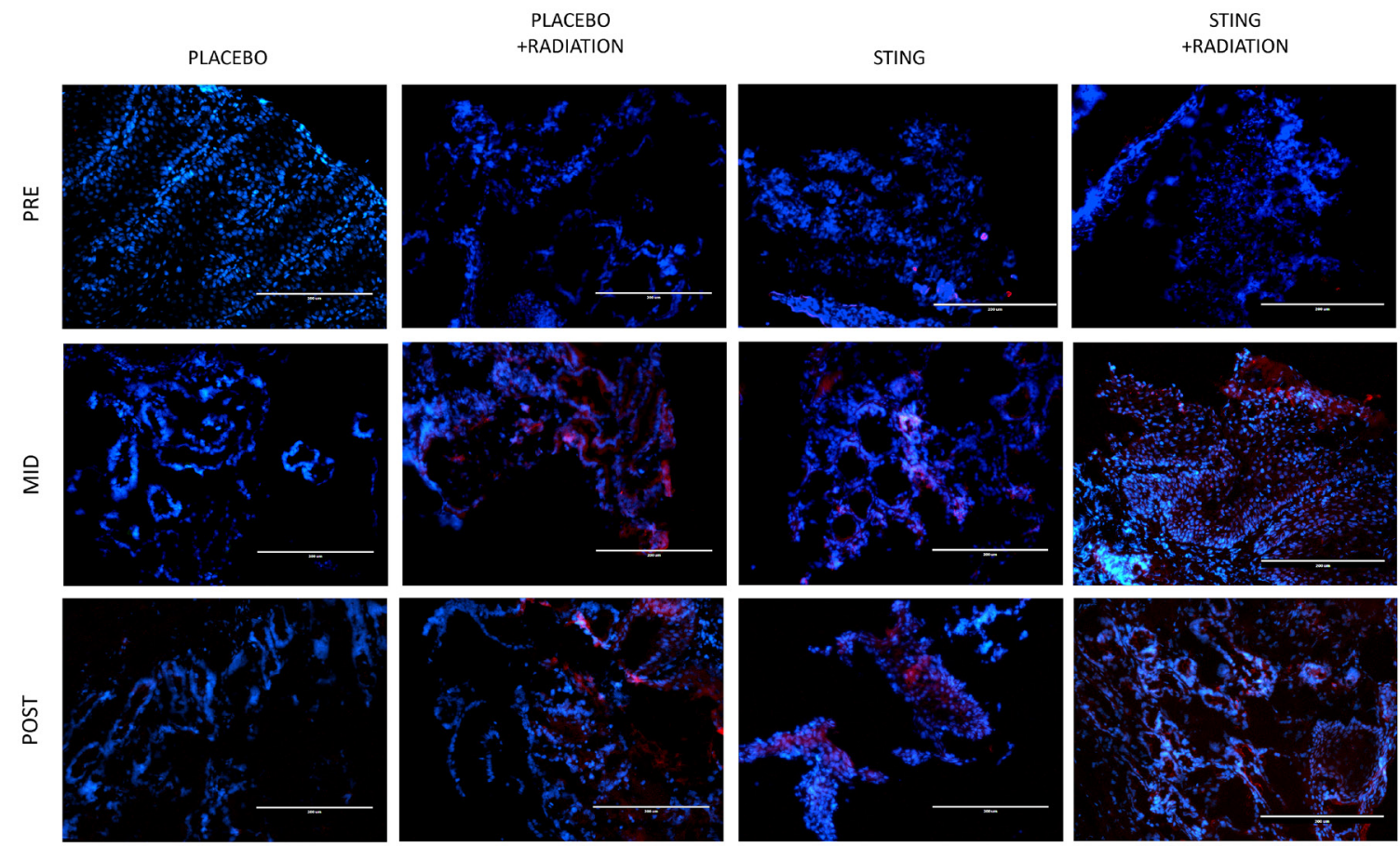

Figure 5: PD-L1 immunofluorescence, by treatment group. Representative study subgroup tumor samples showing membranous PD-L1 immunofluorescence pre-, on- and post-intratumoral injections with placebo or ADU-S100, +/ 16Gy radiation. Positive PDL1 staining was detected at 20x magnification in the primary tumor with the Alexa Fluor 594 secondary antibody, conjugated to a red fluorophore.

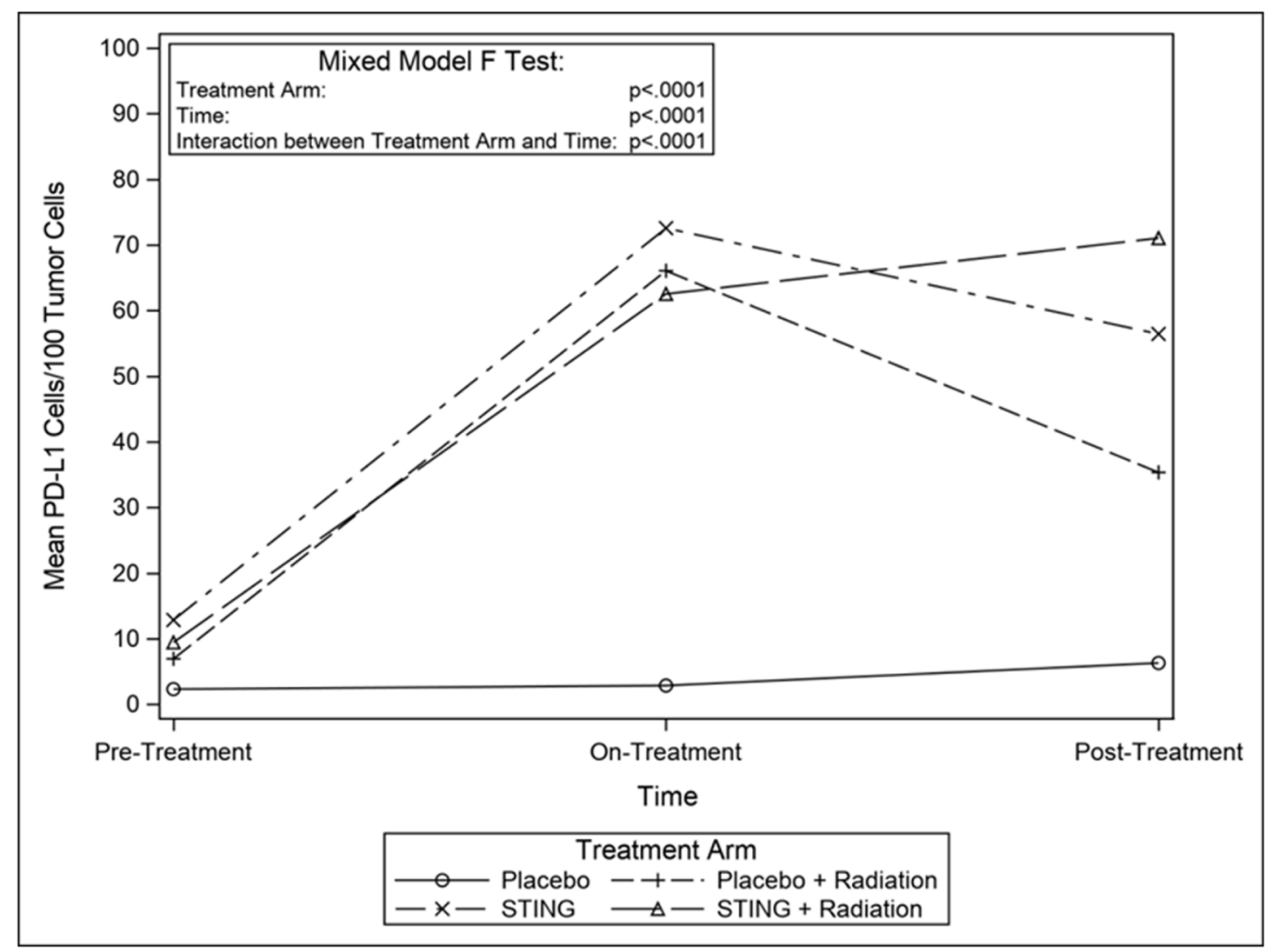

Figure 6: Mean CD8 cells/100 tumor cells, by treatment group. Line graphs depicting changes in mean number of PD-L1 stained cells per 100 tumor cells by study groups pre- on- and post-intervention. 
were administered intratumorally q3 weekly, as 50\% of the total tumor volume. Specifically, administration of the drug was accomplished using a needle through the endoscopic port of a rigid small animal endoscope [35].

\section{Experimental design}

Modified Levrat surgery of end-to-side esophagojejunostomy was performed on 85,8 week old male, Sprague-Dawley rats from Harlan Laboratories, to induce GDER and progression to EAC (Figure 7A), as previously described [19] (Supplementary Figure 1). At 30 weeks post-surgery, all animals received an MRI and endoscopic biopsy of the initial primary tumor to determine initial tumor volume and baseline correlates (Figure 7B). All animals were then randomized into placebo or treatment arms (Figure 8) and if a member of a radiation group, it received one dose of $16 \mathrm{~Gy}$ radiation at 32 weeks [21]. Placebo or ADU-S100 was administered at 32 and 35 weeks intratumorally through endoscopy at a dose of $50 \mu \mathrm{g}$ (Figure 7C). All animals underwent a repeat biopsy at 36 weeks (Figure 7D). At 40 weeks all animals received a final MRI to determine endpoint tumor volume and were euthanized for esophageal harvest.
Activity of ADU-S100 was determined through the comparison of pre and post-treatment tumor volumes of each animal utilizing evaluable MRIs, quantitative realtime polymerase chain reaction (qRT-PCR) analysis of gene expression from serial tissue samples, and evaluation of immunofluorescence for CD8 and PDL1 proteins. MRI imaging response was scored according to the clinical gold standard of response evaluation criteria in solid tumors (RECIST version 1.1) [36].

\section{Tissue preparation}

Pre-, on- and- post-treatment biopsy tissues were flash-frozen in Tissue-Tek Optimal Cutting Temperature (OCT) compound (Sakura Finetek, Torrance, CA, USA; \#4583). Upon necropsy, the entire esophagus and jejunum, to a length approximately $1 \mathrm{~cm}$ distal to the anastomosis was harvested. After the specimen was cut open longitudinally, samples were rinsed in ice-cold phosphate buffered saline to remove debris, oriented to maximize exposure of suspicious areas, and flash-frozen in OCT. Next, frozen OCT blocks with adequate and evaluable tissue were cut into $5 \mu \mathrm{M}$ sections using a cryostat (Fisher Scientific, Waltham, MA, USA; Microm HM 550) and
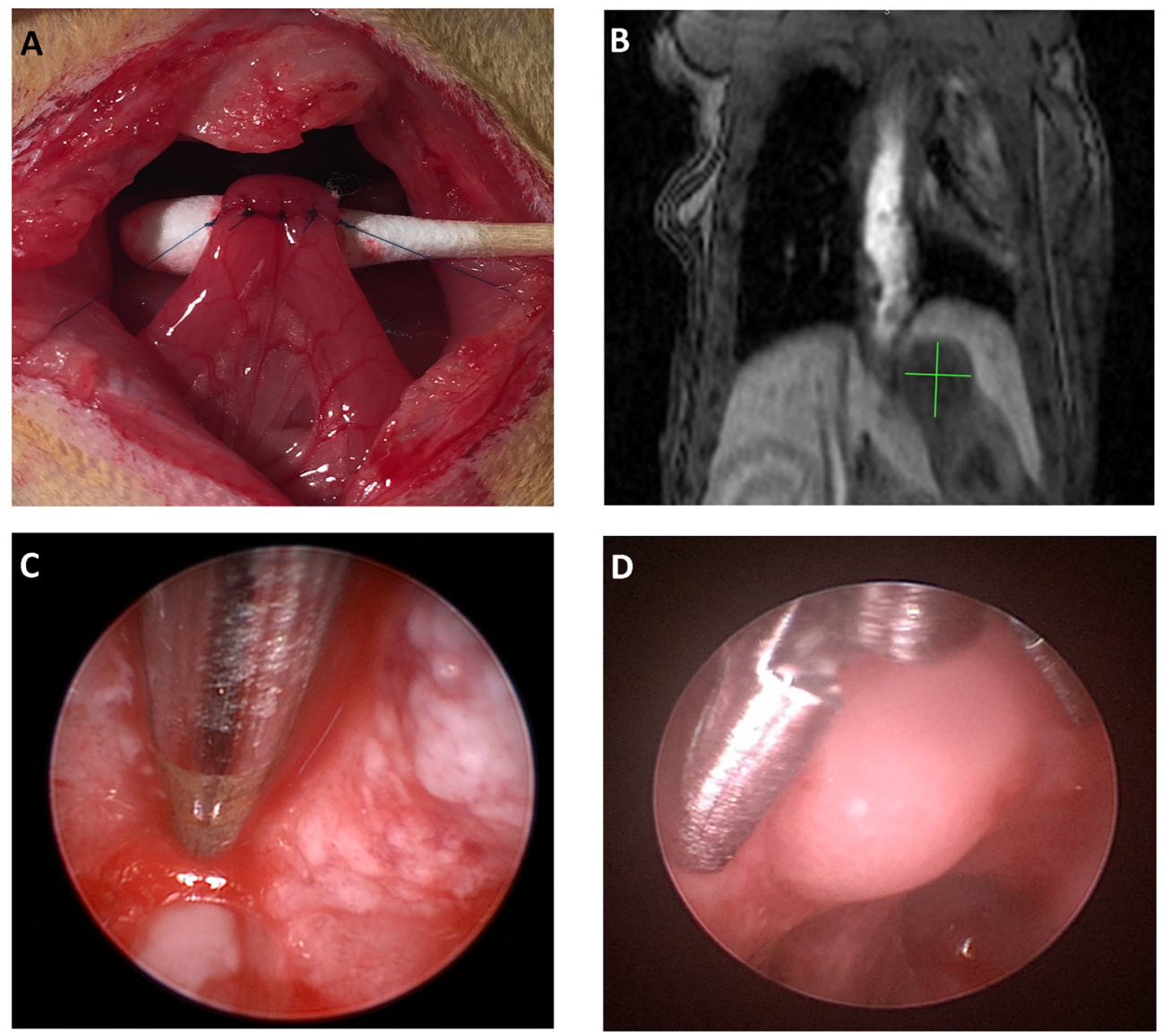

Figure 7: The modified levrat model. (A) End-to-side anastomosis of the esophagus with the jejunum performed as part of the modified Levrat surgery. (B) Coronal MRI image with a visible tumor at the esophagojejunal anastomosis. (C and D) Visual representation of an intratumoral injection and endoscopic biopsy of a suspected tumor in the modified Levrat model, respectively. 
stained with hematoxylin and eosin (H\&E) for pathological assessment, gene expression analysis, and immunoassays.

\section{Gene expression analysis}

A total of $80 \mu \mathrm{M}$ of tissue was macrodissected using a cryostat (Fisher Scientific, Waltham, MA, USA; Microm HM 550). Briefly, RNA was isolated, reverse transcribed, and RT-PCR was performed using rat primers; CCL2 (Qiagen, Valencia, CA, USA, \# PPR06714B), IFN $\beta$ (Qiagen, Valencia, CA, USA, \#PPR06442B), IL6 (Qiagen, Valencia, CA, USA, \# PPR06483B), IFN $\gamma$ (Qiagen, Valencia, CA, USA, \#PPR45050C), and TNFa (Qiagen, Valencia, CA, USA, \#PPR06411F) [37]. Raw data were exported from the realtime instrument software and relative gene expression (RQ) was calculated using the $\Delta \Delta$-Ct method. Specific RT ${ }^{2}$ Primer
Assays Endogenous controls run on each plate included SNORD-95 (Qiagen, Valencia, CA, USA, \#331452), and miR16 (Qiagen, Valencia, CA, USA, \#331452). All samples were normalized against pathologically confirmed normal esophagus and were run in technical duplicates.

\section{Immunofluorescent labeling (IF)}

CD8 and PDL1 antigens were examined using an indirect double sequential immunofluorescence assay. Sample sections were fixated in $4 \%$ paraformaldehyde in PBS solution for $15 \mathrm{~min}$ at room temperature, then washed 3 times in 1xTBS and blocked with 1xTBS containing $5 \%$ goat serum and $1 \%$ BSA for 2 hours. Incubation with anti-CD8 antibody (Abcam, Cambridge, UK; \#ab33786) was performed for 1 hour at 1:100 dilution followed by

\section{STING Agonist, in the Modified Levrat Model: Study Design}
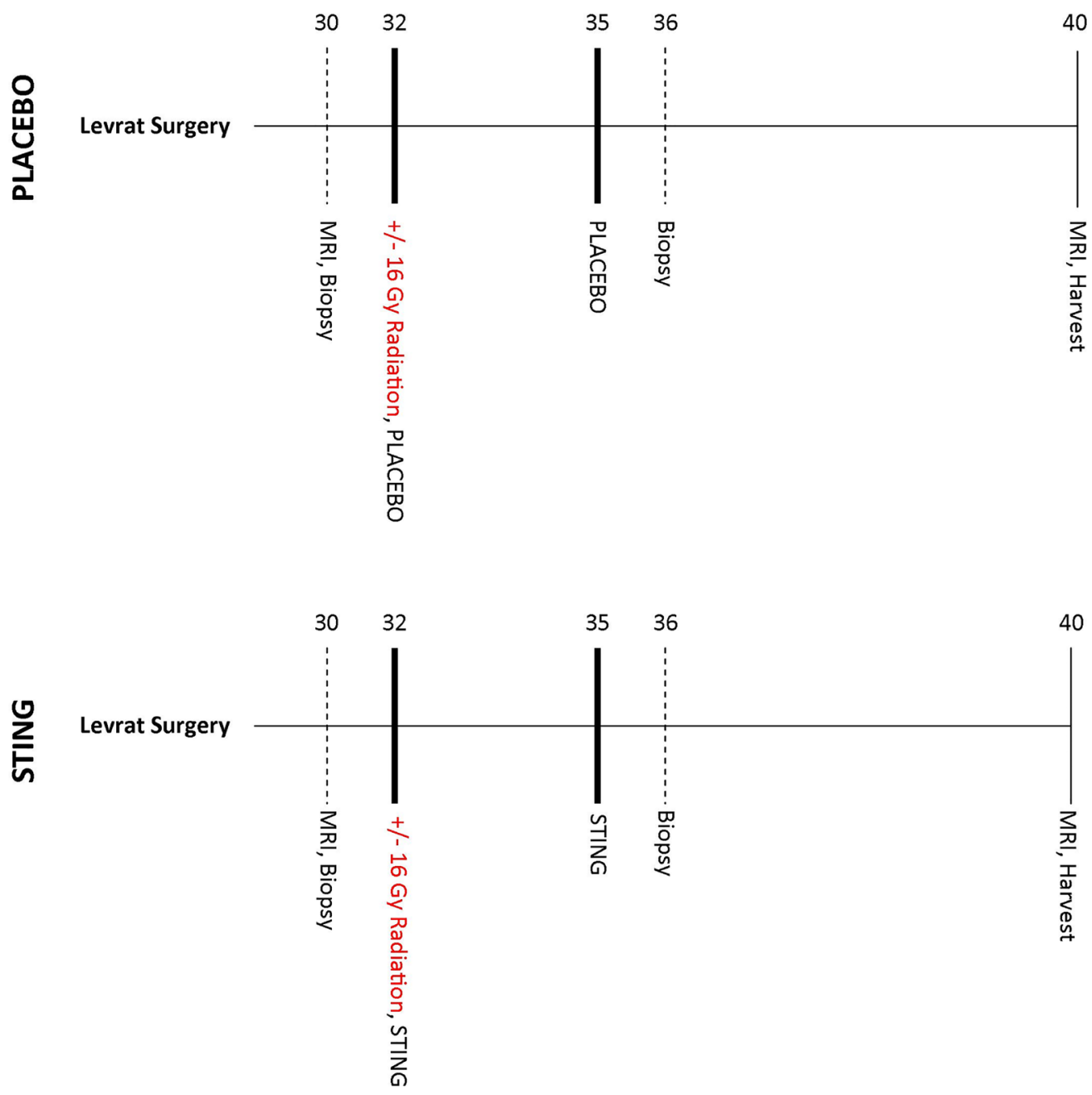

Figure 8: STING study design. Study design outlining the major experimental time points following randomization at 30 weeks post modified Levrat surgery. Starting at 32 weeks animals received 2 cycles of intratumoral injections of placebo or ADU-S100, q3 weekly. In addition if assigned to a radiation subgroup animals received a 16Gy dose of radiation at 32 weeks. All study rats received a final postintervention MRI at 40 weeks followed by a final harvest of the esophagus. 
3 TBS washes containing $0.025 \%$ Tween-20. A second incubation with anti-PDL1 antibody (Thermo Fisher Scientific, Waltham, MA, USA; \#PA5-20343) at 1:100 dilution at $20 \mathrm{ug} / \mathrm{mL}$ for 1 hour was done followed by 3 TBS washes containing $0.025 \%$ Tween-20. Secondary antibody incubation was performed using 1:300 dilutions of goat anti-mouse IgG H\&L Alexa Fluor 488 (Abcam, Cambridge, UK; \#ab150113) and of goat anti-rabbit Alexa 594 (Thermo Fisher Scientific, Waltham, MA, USA; \# A-11037) for anti-CD8 and PDL1, respectively. This was followed by 3 TBST washes. Slides were then rinsed in water and allowed to dry followed by the addition of one drop of Prolong Gold with 4', 6-diamidino-2-phenylindole (DAPI) (Thermo Fisher Scientific, Waltham, MA, USA; \#P36935). After incubation for $24 \mathrm{~h}$ at room temperature in the dark, cells were observed on a fluorescence microscope with light intensity $50 \%$ and exposure time of $60 \mathrm{~ms}$ for DAPI channel, with light intensity $60 \%$ and exposure time of $120 \mathrm{~ms}$ for GFP channel, and with light intensity $70 \%$ and exposure time of $750 \mathrm{~ms}$ for RFP channel.

For PD-L1 and CD8 staining each, a quantitative density analysis was performed for each sample using 3 selected microscopic fields and counting the number of stained cells per 100 tumor cells. Rat lymph node and PDL1+ tumor control tissue served as positive controls for CD8 and PD-L1, respectively. Scoring was performed by three blinded trained research associates with collaborative consensus on cases with discrepant interpretation.

\section{Statistical analysis}

An independent two-tailed $t$ test or WilcoxonMann-Whitney test was utilized for comparison of mean difference between any two treatment groups for CD8 cell density, PD-L1 cell density, and gene expression. ANOVA test was used to compare all groups for percent change of tumor volume. Fisher's exact test was used for comparison of mortality status and change in MRI tumor volume among all treatment groups, respectively. A mixed model F test was performed to evaluate each gene and each protein expression between group effects (treatment group) as well as within subject effects (time point, interaction between treatment group and time point). A $p$ value $<0.05$ was considered to be statistically significant. All statistical analyses were performed using SAS software (version 9.4; SAS Institute, Cary, NC, USA).

\section{Abbreviations}

MSI-H: microsatellite instability high tumors; ICIs: immune checkpoint inhibitors; CAR-T: chimeric antigen receptor T-cells; PD-1: programmed cell death -1 receptor; TIL: tumor-infiltrating T-cells; EC: esophageal cancer; ESCC: esophageal squamous cell carcinoma; CPS: combined positivity score; STING: stimulator of interferon genes; IFN: interferons; DC: dendritic cells;
TBK1: TANK-binding kinase 1; IKK: IкB kinase; IRF3: interferon regulatory factor 3 ; NF- $\kappa \mathrm{B}$ : nuclear factor- $\kappa \mathrm{B}$; STAT6: signal transducer and activator of transcription; NK: natural killer cells; EAC: esophageal adenocarcinoma; GDER: gastroduodenoesophageal reflux disease; qRTPCR: quantitative real-time polymerase chain reaction; OCT: Optimal Cutting Temperature compound; H\&E: hematoxylin and eosin; RQ: relative gene expression; DAPI: Prolong Gold with 4', 6-diamidino-2-phenylindole; $\mathrm{P}$ : placebo group; $\mathrm{P}+\mathrm{R}$ : placebo with radiation group; $\mathrm{S}$ : STING group; $\mathrm{S}+\mathrm{R}$ : STING with radiation group; IO: immune oncology; GEJ: gastroesophageal junction.

\section{Author contributions}

AHZ, RAL, JEK, and BAJ conceptualized the experiments and study design. Methodology was executed out by AHZ, RJK, AG, ANO, MSS, JEK, and BAJ. Investigations were performed by AG, ANO, MSS, PZ, and SL. Data analysis was conducted by AHZ, RJK, AG, ANO, MSS, and PZ. Original manuscript draft was written by AHZ, RJK and PZ, while all authors reviewed and edited the final manuscript. AHZ and BAJ were responsible for the funding acquisition, while radiation resources were provided by SL. Finally, study oversight was provided by AHZ, RJK, and BAJ.

\section{ACKNOWLEDGMENTS}

Aduro Biotech Inc. and Novartis Inc. for providing the study drug under a material transfer agreement. The staff of the Department of Laboratory Animal Research, Allegheny Research Institute for providing support for animal housing, care, and maintenance. Michael A Paskewicz, Mark Biedka, Paul Joseph, Alaina Thomas, Nobel Chowdhury, and Alex Nolte for providing support in conducting defined experimental tasks during the course of the study.

\section{CONFLICTS OF INTEREST}

Authors have no conflicts of interest to declare.

\section{REFERENCES}

1. Bray F, Ferlay J, Soerjomataram I, Siegel RL, Torre LA, Jemal A. Global cancer statistics 2018: GLOBOCAN estimates of incidence and mortality worldwide for 36 cancers in 185 countries. CA Cancer J Clin. 2018; 68:394424. https://doi.org/10.3322/caac.21492. [PubMed]

2. Kelly RJ. Emerging Multimodality Approaches to Treat Localized Esophageal Cancer. J Natl Compr Canc Netw. 2019; 17:1009-14. https://doi.org/10.6004/jnccn.2019.7337. [PubMed]

3. Napier KJ, Scheerer M, Misra S. Esophageal cancer: A Review of epidemiology, pathogenesis, staging workup 
and treatment modalities. World J Gastrointest Oncol. 2014; 6:112-20. https://doi.org/10.4251/wjgo.v6.i5.112. [PubMed]

4. Zou W, Chen L. Inhibitory B7-family molecules in the tumour microenvironment. Nat Rev Immunol. 2008; 8:46777. https://doi.org/10.1038/nri2326. [PubMed]

5. Freeman GJ, Long AJ, Iwai Y, Bourque K, Chernova T, Nishimura H, Fitz LJ, Malenkovich N, Okazaki T, Byrne MC, Horton HF, Fouser L, Carter L, et al. Engagement of the PD-1 immunoinhibitory receptor by a novel B7 family member leads to negative regulation of lymphocyte activation. J Exp Med. 2000; 192:1027-34. https://doi. org/10.1084/jem.192.7.1027. [PubMed]

6. Gajewski TF, Schreiber H, Fu YX. Innate and adaptive immune cells in the tumor microenvironment. Nat Immunol. 2013; 14:1014-22. https://doi.org/10.1038/ ni.2703. [PubMed]

7. Muro K, Chung HC, Shankaran V, Geva R, Catenacci D, Gupta S, Eder JP, Golan T, Le DT, Burtness B, McRee AJ, Lin CC, Pathiraja K, et al. Pembrolizumab for patients with PD-L1-positive advanced gastric cancer (KEYNOTE-012): a multicentre, open-label, phase $1 \mathrm{~b}$ trial. Lancet Oncol. 2016; 17:717-26. https://doi.org/10.1016/S14702045(16)00175-3.

8. Thompson ED, Zahurak M, Murphy A, Cornish T, Cuka N, Abdelfatah E, Yang S, Duncan M, Ahuja N, Taube JM, Anders RA, Kelly RJ. Patterns of PD-L1 expression and CD8 $\mathrm{T}$ cell infiltration in gastric adenocarcinomas and associated immune stroma. Gut. 2017; 66:794-801. https:// doi.org/10.1136/gutjnl-2015-310839. [PubMed]

9. Jing W, McAllister D, Vonderhaar EP, Palen K, Riese MJ, Gershan J, Johnson BD, Dwinell MB. STING agonist inflames the pancreatic cancer immune microenvironment and reduces tumor burden in mouse models. J Immunother Cancer. 2019; 7:115. https://doi.org/10.1186/s40425-0190573-5. [PubMed]

10. Flood BA, Higgs EF, Li S, Luke JJ, Gajewski TF. STING pathway agonism as a cancer therapeutic. Immunol Rev. 2019; 290:24-38. https://doi.org/10.1111/imr.12765. [PubMed]

11. Paludan SR, Bowie AG. Immune sensing of DNA. Immunity. 2013; 38:870-80. https://doi.org/10.1016/j. immuni.2013.05.004. [PubMed]

12. Fuertes MB, Woo SR, Burnett B, Fu YX, Gajewski TF. Type I interferon response and innate immune sensing of cancer. Trends Immunol. 2013; 34:67-73. https://doi.org/10.1016/j. it.2012.10.004. [PubMed]

13. Corrales L, Glickman LH, McWhirter SM, Kanne DB, Sivick KE, Katibah GE, Woo SR, Lemmens E, Banda T, Leong JJ, Metchette K, Dubensky TW Jr, Gajewski TF. Direct Activation of STING in the Tumor Microenvironment Leads to Potent and Systemic Tumor Regression and Immunity. Cell Rep. 2015; 11:1018-30. https://doi.org/10.1016/j.celrep.2015.04.031. [PubMed]
14. Ohkuri T, Kosaka A, Ishibashi K, Kumai T, Hirata Y, Ohara K, Nagato T, Oikawa K, Aoki N, Harabuchi Y, Celis E, Kobayashi $H$. Intratumoral administration of cGAMP transiently accumulates potent macrophages for antitumor immunity at a mouse tumor site. Cancer Immunol Immunother. 2017; 66:705-16. https://doi.org/10.1007/ s00262-017-1975-1. [PubMed]

15. Liang H, Deng L, Hou Y, Meng X, Huang X, Rao E, Zheng W, Mauceri H, Mack M, Xu M, Fu YX, Weichselbaum RR. Host STING-dependent MDSC mobilization drives extrinsic radiation resistance. Nat Commun. 2017; 8:1736. https://doi.org/10.1038/s41467-017-01566-5. [PubMed]

16. Gadkaree SK, Fu J, Sen R, Korrer MJ, Allen C, Kim YJ. Induction of tumor regression by intratumoral STING agonists combined with anti-programmed death-L1 blocking antibody in a preclinical squamous cell carcinoma model. Head Neck. 2017; 39:1086-94. https://doi. org/10.1002/hed.24704. [PubMed]

17. Deng L, Liang H, Xu M, Yang X, Burnette B, Arina A, Li XD, Mauceri H, Beckett M, Darga T, Huang X, Gajewski TF, Chen ZJ, et al. STING-Dependent Cytosolic DNA Sensing Promotes Radiation-Induced Type I InterferonDependent Antitumor Immunity in Immunogenic Tumors. Immunity. 2014; 41:843-52. https://doi.org/10.1016/j. immuni.2014.10.019. [PubMed]

18. Motedayen Aval L, Pease JE, Sharma R, Pinato DJ. Challenges and Opportunities in the Clinical Development of STING Agonists for Cancer Immunotherapy. J Clin Med. 2020; 9:3323. https://doi.org/10.3390/jcm9103323. [PubMed]

19. Gibson MK, Zaidi AH, Davison JM, Sanz AF, Hough B, Komatsu Y, Kosovec JE, Bhatt A, Malhotra U, Foxwell T, Rotoloni CL, Hoppo T, Jobe BA. Prevention of Barrett esophagus and esophageal adenocarcinoma by smoothened inhibitor in a rat model of gastroesophageal reflux disease. Ann Surg. 2013; 258:82-8. https://doi.org/10.1097/ SLA.0b013e318270500d. [PubMed]

20. Zaidi AH, Saldin LT, Kelly LA, Bergal L, Londono R, Kosovec JE, Komatsu Y, Kasi PM, Shetty AA, Keane TJ, Thakkar SJ, Huleihel L, Landreneau RJ, et al. MicroRNA signature characterizes primary tumors that metastasize in an esophageal adenocarcinoma rat model. PLoS One. 2015; 10:e0122375. https://doi.org/10.1371/journal.pone.0122375. [ [PubMed]

21. Kelly RJ, Zaidi AH, Smith MA, Omstead AN, Kosovec JE, Matsui D, Martin SA, DiCarlo C, Werts ED, Silverman JF, Wang DH, Jobe BA. The Dynamic and Transient Immune Microenvironment in Locally Advanced Esophageal Adenocarcinoma Post Chemoradiation. Ann Surg. 2018; 268:992-9. https://doi.org/10.1097/ SLA.0000000000002410. [PubMed]

22. Fu J, Kanne DB, Leong M, Glickman LH, McWhirter SM, Lemmens E, Mechette K, Leong JJ, Lauer P, Liu W, Sivick $\mathrm{KE}$, Zeng Q, Soares KC, et al. STING agonist formulated cancer vaccines can cure established tumors resistant to PD-1 blockade. Sci Transl Med. 2015; 7:283ra52. https:// doi.org/10.1126/scitranslmed.aaa4306. [PubMed] 
23. Vanpouille-Box C, Demaria S, Formenti SC, Galluzzi L. Cytosolic DNA Sensing in Organismal Tumor Control. Cancer Cell. 2018; 34:361-78. https://doi.org/10.1016/j. ccell.2018.05.013. [PubMed]

24. Chattopadhyay S, Marques JT, Yamashita M, Peters KL, Smith K, Desai A, Williams BR, Sen GC. Viral apoptosis is induced by IRF-3-mediated activation of Bax. EMBO J. 2010; 29:1762-73. https://doi.org/10.1038/emboj.2010.50. [PubMed]

25. Sivick KE, Desbien AL, Glickman LH, Reiner GL, Corrales L, Surh NH, Hudson TE, Vu UT, Francica BJ, Banda T, Katibah GE, Kanne DB, Leong JJ, et al. Magnitude of Therapeutic STING Activation Determines CD8(+) T CellMediated Anti-tumor Immunity. Cell Rep. 2018; 25:307485.e5. https://doi.org/10.1016/j.celrep.2018.11.047. [PubMed]

26. Woo SR, Corrales L, Gajewski TF. The STING pathway and the $\mathrm{T}$ cell-inflamed tumor microenvironment. Trends Immunol. 2015; 36:250-6. https://doi.org/10.1016/j. it.2015.02.003. [PubMed]

27. Kang YK, Boku N, Satoh T, Ryu MH, Chao Y, Kato K, Chung HC, Chen JS, Muro K, Kang WK, Yeh KH, Yoshikawa T, Oh SC, et al. Nivolumab in patients with advanced gastric or gastro-oesophageal junction cancer refractory to, or intolerant of, at least two previous chemotherapy regimens (ONO-4538-12, ATTRACTION-2): a randomised, double-blind, placebo-controlled, phase 3 trial. Lancet. 2017; 390:2461-71. https://doi.org/10.1016/ S0140-6736(17)31827-5.

28. Fuchs CS, Doi T, Jang RW, Muro K, Satoh T, Machado M, Sun W, Jalal SI, Shah MA, Metges JP, Garrido M, Golan T, Mandala M, et al. Safety and Efficacy of Pembrolizumab Monotherapy in Patients With Previously Treated Advanced Gastric and Gastroesophageal Junction Cancer: Phase 2 Clinical KEYNOTE-059 Trial. JAMA Oncol. 2018; 4:e180013. https://doi.org/10.1001/jamaoncol.2018.0013. [PubMed]

29. Kojima T, Muro K, Francois E, Hsu CH, Moriwaki T, Kim SB, Lee SH, Bennouna J, Kato K, Lin S, Qin SQ, Ferreira $\mathrm{P}$, Doi T, et al. Pembrolizumab versus chemotherapy as second-line therapy for advanced esophageal cancer: Phase III KEYNOTE-181 study. J Clin Oncol. 2019; 37:2. https:// doi.org/10.1200/JCO.2019.37.4 suppl.2.

30. Shah MA, Kojima T, Hochhauser D, Enzinger P, Raimbourg J, Hollebecque A, Lordick F, Kim SB, Tajika M, Kim HT, Lockhart AC, Arkenau HT, El-Hajbi F, et al. Efficacy and Safety of Pembrolizumab for Heavily Pretreated Patients With Advanced, Metastatic Adenocarcinoma or Squamous Cell Carcinoma of the Esophagus: The Phase 2 KEYNOTE-180 Study. JAMA Oncol. 2019; 5:546-50. https://doi.org/10.1001/jamaoncol.2018.5441. [PubMed]
31. Tabernero J, Cutsem EV, Bang YJ, Fuchs CS, Wyrwicz L, Lee KW, Kudaba I, Garrido M, Chung HC, Salguero HRC, Mansoor W, Braghiroli MIFM, Goekkurt E, et al. Pembrolizumab with or without chemotherapy versus chemotherapy for advanced gastric or gastroesophageal junction (G/GEJ) adenocarcinoma: The phase III KEYNOTE-062 study. J Clin Oncol. 2019; 37:LBA4007LBA. $\quad$ https://doi.org/10.1200/JCO.2019.37.18 suppl. $\underline{\text { LBA4007. }}$.

32. Janjigian YY, Bendell J, Calvo E, Kim JW, Ascierto PA, Sharma P, Ott PA, Peltola K, Jaeger D, Evans J, de Braud F, Chau I, Harbison CT, et al. CheckMate-032 Study: Efficacy and Safety of Nivolumab and Nivolumab Plus Ipilimumab in Patients With Metastatic Esophagogastric Cancer. J Clin Oncol. 2018; 36:2836-44. https://doi.org/10.1200/ JCO.2017.76.6212. [PubMed]

33. Walsh EM, Kelly RJ. Single agent anti PD-1 inhibitors in esophageal cancer-a first step in a new therapeutic direction. J Thorac Dis. 2018; 10:1308-13. https://doi.org/10.21037/ jtd.2018.03.43. [PubMed]

34. Moore E, Clavijo PE, Davis R, Cash H, Van Waes C, Kim Y, Allen C. Established T Cell-Inflamed Tumors Rejected after Adaptive Resistance Was Reversed by Combination STING Activation and PD-1 Pathway Blockade. Cancer Immunol Res. 2016; 4:1061-71. https://doi.org/10.1158/2326-6066. CIR-16-0104. [PubMed]

35. Omstead AN, Kosovec JE, Matsui D, Martin SA, Smith MA, Aaron Guel D, Kolano J, Komatsu Y, Habib F, Lai C, Christopher K, Kelly RJ, Zaidi AH, et al. Serial Endoscopic Evaluation of Esophageal Disease in a Cancer Model: A Paradigm Shift for Esophageal Adenocarcinoma (EAC) Drug Discovery and Development. Cancer Invest. 2018; 36:363-70. https://doi.org/10.1080/07357907.2018.14990 29. [PubMed]

36. Eisenhauer EA, Therasse P, Bogaerts J, Schwartz LH, Sargent D, Ford R, Dancey J, Arbuck S, Gwyther S, Mooney M, Rubinstein L, Shankar L, Dodd L, et al. New response evaluation criteria in solid tumours: revised RECIST guideline (version 1.1). Eur J Cancer. 2009; 45:228-47. https://doi.org/10.1016/j.ejca.2008.10.026. [PubMed]

37. Kosovec JE, Zaidi AH, Kelly LA, Rotoloni CL, Vytlacil C, DiCarlo C, Matsui D, Komatsu Y, Boyd NH, Omstead A, Kolano EL, Biederman RW, Finley G, et al. Preclinical Study of AUY922, a Novel Hsp90 Inhibitor, in the Treatment of Esophageal Adenocarcinoma. Ann Surg. 2016; 264:297-304. https://doi.org/10.1097/ SLA.0000000000001467. [PubMed] 